\title{
On Partitioning and Subtractive Subsemimodules of Semi- modules over Semirings
}

\author{
Jaiprakash Ninu Chaudhari* And DipaK RAvindRa Bonde \\ Department of Mathematics, Moolji Jaitha College, Jalgaon-425002, India \\ e-mail : jnchaudhari@rediffmail.com and drbonde@rediffmail.com
}

ABstract. In this paper, we introduce a partitioning subsemimodule of a semimodule over a semiring which is useful to develop the quotient structure of semimodule. Indeed we prove : 1) The quotient semimodule $M / N_{(Q)}$ is essentially independent of choice of $Q$. 2) If $f: M \rightarrow M^{\prime}$ is a maximal $R$-semimodule homomorphism, then $M / \operatorname{ker} f_{(Q)} \cong M^{\prime} .3$ ) Every partitioning subsemimodule is subtractive. 4) Let $N$ be a $Q$-subsemimodule of an $R$-semimodule $M$. Then $A$ is a subtractive subsemimodule of $M$ with $N \subseteq A$ if and only if $A / N_{(Q \cap A)}=\{q+N: q \in Q \cap A\}$ is a subtractive subsemimodule of $M / N_{(Q)}$.

\section{Introduction}

For the definitions of monoid and semiring we refer [5 ]. All semirings in this paper are commutative with identity element. $\mathbb{Z}_{0}^{+}$will denote the set of all nonnegative integers. An element $a$ of a monoid $(M, *)$ is called idempotent if $a * a=a$. An ideal $I$ of a semiring $R$ is called a subtractive ideal ( $k$-ideal) if $a, a+b \in I, b \in R$, then $b \in I$. An ideal $I$ of a semiring $R$ is called a partitioning ideal (=Q-ideal) if there exist a subset $Q$ of $R$ such that:

1) $R=\cup\{q+I: q \in Q\}$.

2) If $q_{1}, q_{2} \in Q$, then $\left(q_{1}+I\right) \cap\left(q_{2}+I\right) \neq \emptyset \Leftrightarrow q_{1}=q_{2}$.

Definition 1.1. Let $R$ be a semiring. A left $R$-semimodule is a commutative monoid $(M,+)$ with additive identity $0_{M}$ for which we have a function $R \times M \rightarrow M$, defined by $(r, x) \mapsto r x$ called scalar multiplication, which satisfies the following conditions for all elements $r$ and $r^{\prime}$ of $R$ and all elements $x$ and $y$ of $M$ :

1) $\left(r r^{\prime}\right) x=r\left(r^{\prime} x\right)$;

2) $r(x+y)=r x+r y$

3) $\left(r+r^{\prime}\right) x=r x+r^{\prime} x$;

4) $1_{R} x=x$

* Corresponding Author.

Received December 2, 2009; revised May 10, 2010; accepted May 14, 2010.

2000 Mathematics Subject Classification: 16Y60.

Key words and phrases: semimodule; subtractive subsemimodule; partitioning subsemimodule; quotient semimodule; maximal homomorphism; isomorphism. 
5) $r 0_{M}=0_{M}=0_{R} x$.

A nonempty subset $N$ of a left $R$-semimodule $M$ is called subsemimodule of $M$ if $N$ is closed under addition and closed under scalar multiplication.

Throughout this paper by an $R$-semimodule we mean a left semimodule over a semiring $R$. Every semiring $R$ is $\left(\mathbb{Z}_{0}^{+},+, \cdot\right)$-semimodule ([5], P.151).

Definition 1.2. If $R$ is a semiring and $M$ and $M^{\prime}$ are $R$-semimodules, then a function $f$ from $M$ to $M^{\prime}$ is an $R$-semimodule homomorphism if and only if the following conditions are satisfied:

1) $f(x+y)=f(x)+f(y)$ for all $x, y \in M$;

2) $f(r x)=r f(x)$ for all $x \in M$ and $r \in R$.

An $R$-semimodule homomorphism $f$ from an $R$-semimodule $M$ to an $R$-semimodule $M^{\prime}$ is called isomorphism if $f$ is one to one and onto.

The following lemma which is similar to lemma([6], Lemma 1) is easy to prove.

Lemma 1.3. Let $N$ be a subsemimodule of an $R$-semimodule $M$ and $x, y \in M$ such that $x+N \subseteq y+N$. Then $x+z+N \subseteq y+z+N$ and $r x+N \subseteq r y+$ $N$ for all $z \in M, r \in R$.

\section{Partitioning Subsemimodules}

In this section we extend some definitions and results of Allen [1], [2] and Atani [3] to semimodules over semirings.

Definition 2.1. A subsemimodule $N$ of an $R$-semimodule $M$ will be called a partitioning subsemimodule (= $Q$-subsemimodule) if there exists a subset $Q$ of $M$ such that

1) $M=\cup\{q+N: q \in Q\}$.

2) if $q_{1}, q_{2} \in Q$, then $\left(q_{1}+N\right) \cap\left(q_{2}+N\right) \neq \emptyset \Leftrightarrow q_{1}=q_{2}$.

Clearly, every semiring is semimodule over itself. Hence every partitioning ideal of a semiring $R$ is partitioning subsemimodule of an $R$-semimodule $R$.

Lemma 2.2. Let $N$ be a partitioning subsemimodule of an $R$-semimodule $M$. If $x \in M$, then there exists a unique $q \in Q$ such that $x+N \subseteq q+N$. Hence $x=q+$ a for some $a \in N$.

Proof. Trivial.

Now we extend a result of P. J. Allen ([2], Lemma 36) for semirings to semimodules over semirings.

Lemma 2.3. If $N$ is a partitioning subsemimodule of an $R$-semimodule $M$, then 
there exists a unique $q_{0} \in Q$ such that $N=q_{0}+N$.

Proof. Since $N$ is a partitioning subsemimodule, by Lemma 2.2, there exists a unique $q_{0} \in Q$ such that $0=q_{0}+a_{0}$ for some $a_{0} \in N$. If $b \in N$, then by Lemma 2.2 , there exists a unique $q \in Q$ such that $b=q+a$ for some $a \in N$. Therefore, $q$ $+a=b=b+0=b+q_{0}+a_{0} \in q_{0}+N$. Hence $N \subseteq q_{0}+N$. Again by Lemma 2.2 , there exists a unique $q^{\prime} \in Q$ such that $q_{0}+q_{0}=q^{\prime}+c$ for some $c \in N$. Now $q_{0}=q_{0}+0=q_{0}+q_{0}+a_{0}=q^{\prime}+c+a_{0} \in q^{\prime}+N$. Also $q_{0} \in q_{0}+N$. Hence $\left(q^{\prime}\right.$ $+N) \cap\left(q_{0}+N\right) \neq \emptyset$ and so $q_{0}=q^{\prime}$. Thus, $q_{0}+N=q^{\prime}+c+a_{0}+N=q_{0}+c$ $+a_{0}+N=c+q_{0}+a_{0}+N=c+N \subseteq N$. Now $N=q_{0}+N$ where $q_{0} \in Q$ is a unique element.

Let $N$ be a partitioning subsemimodule of an $R$-semimodule $M$. Then $M / N_{(Q)}$ $=\{q+N: q \in Q\}$ forms an $R$-semimodule under the following addition " $\oplus$ " and scalar multiplication " $\odot$ ", $\left(q_{1}+N\right) \oplus\left(q_{2}+N\right)=q_{3}+N$ where $q_{3} \in Q$ is a unique element such that $q_{1}+q_{2}+N \subseteq q_{3}+N$ and $r \odot\left(q_{1}+N\right)=q_{4}+N$ where $q_{4} \in$ $Q$ is a unique element such that $r q_{1}+N \subseteq q_{4}+N$. This $R$-semimodule $M / N_{(Q)}$ will be called a quotient semimodule of $M$ by $N$ and denoted by $\left(M / N_{(Q)}, \oplus, \odot\right)$ or just $M / N_{(Q)}$. By Lemma 2.3, there exists a unique $q_{0} \in Q$ such that $q_{0}+N$ $=N$. This $q_{0}+N$ is the zero element of $M / N_{(Q)}$. If $N$ is a subsemimodule of an $R$-semimodule $M$, then it is possible that $N$ can be considered to be a partitioning subsemimodule with respet to many different subsets $Q$ of $M$. However, the next theorem proves that the structure $\left(M / N_{(Q)}, \oplus, \odot\right)$ is essentially independent of $Q$.

Theorem 2.4. If $N$ is a partitioning subsemimodule with respect to two subsets $Q_{1}$ and $Q_{2}$ of an $R$-semimodule $M$, then $M / N_{\left(Q_{1}\right)} \cong M / N_{\left(Q_{2}\right)}$.

Proof. Define $f: M / N_{\left(Q_{1}\right)} \rightarrow M / N_{\left(Q_{2}\right)}$ by $f\left(q_{1}+N\right)=q_{2}+N$ where $q_{2} \in Q_{2}$ is a unique such that $q_{1}+N \subseteq q_{2}+N$. Clearly, $f$ is well defined.

1) Let $q_{1}+N, q_{1}^{\prime}+N \in M / N_{\left(Q_{1}\right)}$ and $r \in R$. Therefore,

$$
f\left(\left(q_{1}+N\right) \oplus\left(q_{1}{ }^{\prime}+N\right)\right)=f\left(q_{1}{ }^{\prime \prime}+N\right)=q_{2}+N
$$

where $q_{1}{ }^{\prime \prime} \in Q_{1}$ is a unique such that $q_{1}+q_{1}{ }^{\prime}+N \subseteq q_{1}{ }^{\prime \prime}+N$ and $q_{2} \in Q_{2}$ is a unique such that $q_{1}^{\prime \prime}+N \subseteq q_{2}+N$. Also

$$
f\left(q_{1}+N\right) \oplus f\left(q_{1}{ }^{\prime}+N\right)=\left(q_{2}{ }^{\prime}+N\right) \oplus\left(q_{2}{ }^{\prime \prime}+N\right)=q_{2}{ }^{\prime \prime \prime}+N
$$

where $q_{2}{ }^{\prime},{ }_{\prime \prime \prime} q_{2}{ }^{\prime \prime} \in Q_{2}$ are unique such that $q_{1}+\underset{\prime \prime}{N} \subseteq q_{2}{ }^{\prime}+N_{\prime \prime \prime}$ and $q_{1}{ }^{\prime}+N \subseteq q_{2}{ }^{\prime \prime}+$ $N$ and $q_{2}^{\prime \prime \prime} \in Q_{2}$ is a unique such that $q_{2}^{\prime}+q_{2}^{\prime \prime}+N \subseteq q_{2}^{\prime \prime \prime}+N$. Now

$$
q_{1}+q_{1}{ }^{\prime} \in q_{1}+q_{1}{ }^{\prime}+N \subseteq q_{1}{ }^{\prime \prime}+N \subseteq q_{2}+N .
$$

Also by Lemma 1.3,

$$
\begin{aligned}
q_{1}+N \subseteq q_{2}{ }^{\prime}+N \text { and } \quad q_{1}{ }^{\prime}+N \subseteq q_{2}{ }^{\prime \prime}+N \Rightarrow q_{1}+q_{1}{ }^{\prime}+N & \subseteq q_{2}{ }^{\prime}+q_{1}{ }^{\prime}+N \\
& \subseteq q_{2}{ }^{\prime}+q_{2}{ }^{\prime \prime}+N \\
& \subseteq q_{2}{ }^{\prime \prime \prime}+N .
\end{aligned}
$$


Therefore,

$$
q_{1}+q_{1}^{\prime} \in q_{1}+q_{1}{ }^{\prime}+N \subseteq q_{2}^{\prime \prime \prime}+N
$$

From (iii) and (iv), $q_{2}=q_{2}{ }^{\prime \prime \prime}$. Hence by (i) and (ii), $f\left(\left(q_{1}+N\right) \oplus\left(q_{1}{ }^{\prime}+N\right)\right)$ $=f\left(q_{1}+N\right) \oplus f\left(q_{1}{ }^{\prime}+N\right)$. Similarly, it can be shown that $f\left(r \odot\left(q_{1}+N\right)\right)=$ $r \odot f\left(q_{1}+N\right)$.

2) Let $q_{2}+N \in M / N_{\left(Q_{2}\right)}$. Since $q_{2} \in M$, there exists a unique $q_{1} \in Q_{1}$ such that $q_{2}+N \subseteq q_{1}+N$. But then there exists a unique $q_{2}{ }^{\prime} \in Q_{2}$ such that $q_{1}+N \subseteq$ $q_{2}{ }^{\prime}+N$. Now $q_{2}=q_{2}{ }^{\prime}$ implies $q_{2}+N=q_{2}{ }^{\prime}+N$ and hence $f\left(q_{1}+N\right)=q_{2}+N$. So $f$ is onto.

3) Suppose that $f\left(q_{1}+N\right)=f\left(q_{1}{ }^{\prime}+N\right)=q_{2}+N$ say, where $q_{2} \in Q_{2}$ is a unique such that $q_{1}+N \subseteq q_{2}+N$ and $q_{1}^{\prime}+N \subseteq q_{2}+N$. Choose $t_{1} \in Q_{1}$ such that $q_{2}+N \subseteq t_{1}+N$. But then $q_{1}=t_{1}=q_{1}{ }^{\prime}$. So $q_{1}+N=q_{1}{ }^{\prime}+N$. Thus $f: M / N_{\left(Q_{1}\right)} \rightarrow M / N_{\left(Q_{2}\right)}$ is an isomorphism.

Theorem 2.5. If $N$ is a partitioning subsemimodule with respect to two subsets $Q_{1}$ and $Q_{2}$ of an R-semimodule $M$, then $M / N_{\left(Q_{1}\right)}$ and $M / N_{\left(Q_{2}\right)}$ are equal as sets.

Proof. Let $q_{1}+N \in M / N_{\left(Q_{1}\right)}$. Then $q_{1} \in Q_{1} \subseteq M$ and hence by Lemma 2.2, there exists a unique $q_{2} \in Q_{2}$ such that $q_{1}+N \subseteq q_{2}+N$. Again there exists a unique $q_{3} \in Q_{1}$ such that $q_{2}+N \subseteq q_{3}+N$. Now $q_{1}+N=q_{3}+N=q_{2}+N \in M / N_{\left(Q_{2}\right)}$. So $M / N_{\left(Q_{1}\right)} \subseteq M / N_{\left(Q_{2}\right)}$. Similarly, $M / N_{\left(Q_{2}\right)} \subseteq M / N_{\left(Q_{1}\right)}$.

Example 2.6. The monoid $M=\left(\mathbb{Z}_{6},+_{6}\right)$ is semimodule over $\left(\mathbb{Z}_{0}^{+},+, \cdot\right)$, ([5], P.151). Then clearly $N=\{\overline{0}, \overline{2}, \overline{4}\}$ is a partitioning subsemimodule of $M$ with respect to three sets $Q_{1}=\{\overline{0}, \overline{1}\}, Q_{2}=\{\overline{0}, \overline{3}\}, Q_{3}=\{\overline{0}, \overline{5}\}$ where $M / N_{\left(Q_{1}\right)}=\{\overline{0}+$ $N, \overline{1}+N\}=\{\{\overline{0}, \overline{2}, \overline{4}\},\{\overline{1}, \overline{3}, \overline{5}\}\}, M / N_{\left(Q_{2}\right)}=\{\overline{0}+N, \overline{3}+N\}=\{\{\overline{0}, \overline{2}, \overline{4}\},\{\overline{1}, \overline{3}, \overline{5}\}\}$ and $M / N_{\left(Q_{3}\right)}=\{\overline{0}+N, \overline{5}+N\}=\{\{\overline{0}, \overline{2}, \overline{4}\},\{\overline{1}, \overline{3}, \overline{5}\}\}$. Here $M / N_{\left(Q_{1}\right)}, M / N_{\left(Q_{2}\right)}$ and $M / N_{\left(Q_{3}\right)}$ are equal as sets. But $M / N_{\left(Q_{1}\right)}, M / N_{\left(Q_{2}\right)}$ and $M / N_{\left(Q_{3}\right)}$ considered as $\left(\mathbb{Z}_{0}^{+},+, \cdot\right)$-semimodules are not equal because $\overline{1}+N \in M / N_{\left(Q_{1}\right)}$ but $\overline{1}+N \notin$ $M / N_{\left(Q_{2}\right)}$ and $\overline{1}+N \notin M / N_{\left(Q_{3}\right)}$ as $\overline{1} \notin Q_{2}$ and $\overline{1} \notin Q_{3}$.

Definition 2.7. An onto $R$-semimodule homomorphism $f: M \rightarrow M^{\prime}$ will be called maximal if for each $a \in M^{\prime}$ there exists a unique $q_{a} \in f^{-1}(\{a\})$ such that $x+\operatorname{ker} f$ $\subseteq q_{a}+\operatorname{kerf}$, for each $x \in f^{-1}(\{a\})$ where $\operatorname{kerf}=\left\{x \in M: f(x)=0_{M^{\prime}}\right\}$.

Clearly every $R$-module homomorphism is a maximal $R$-semimodule homomorphism.

P. J. Allen ([1], Lemma 14, Lemma 15 and Theorem 16) has proved the results for semirings. However, we extend the following Lemma 2.8, Lemma 2.11 and Theorem 2.12 for semimodules over semirings.

Lemma 2.8. If $f: M \rightarrow M^{\prime}$ is a maximal R-semimodule homomorphism, then kerf is a partitioning subsemimodule of $M$.

Proof. Since $f$ is maximal, for each $a \in M^{\prime}$ there exists a unique $q_{a} \in f^{-1}(\{a\})$ 
such that $x+\operatorname{ker} f \subseteq q_{a}+\operatorname{kerf}$ for all $x \in f^{-1}(\{a\})$. Take $Q=\left\{q_{a}: a \in M^{\prime}\right\}$. Clearly $\cup\left\{q_{a}+\operatorname{kerf}: q_{a} \in Q\right\} \subseteq M$. On the other hand, if $m \in M$, then $f(m) \in$ $M^{\prime}$. Now $m \in f^{-1}(\{f(m)\})$ implies $m \in m+k e r f \subseteq q_{f(m)}+\operatorname{kerf}$. Hence $M \subseteq$ $\cup\left\{q_{a}+\operatorname{kerf}: q_{a} \in Q\right\}$. Now for $q_{a}, q_{b} \in Q$, suppose that $\left(q_{a}+\operatorname{kerf}\right) \cap\left(q_{b}+\operatorname{ker} f\right)$ $\neq \emptyset$. Let $q_{a}+k=q_{b}+k^{\prime}$ for some $k, k^{\prime} \in \operatorname{ker} f$. Now $a=f\left(q_{a}\right)+f(k)=f\left(q_{a}+k\right)$ $=f\left(q_{b}+k^{\prime}\right)=f\left(q_{b}\right)+f\left(k^{\prime}\right)=b$. Hence $q_{a}=q_{b}$. Thus, ker $f$ is a partitioning subsemimodule of $M$.

The converse of Lemma 2.8 is not true.

Example 2.9. Let $M=\left(\mathbb{Z}_{0}{ }^{+}, \max \right), M^{\prime}=(\{0,1\}, \max )$ and $R=\left(\mathbb{Z}_{0}^{+},+, \cdot\right)$. Then $M, M^{\prime}$ are $R$-semimodules. Define $f: M \rightarrow M^{\prime}$ by

$$
f(x)= \begin{cases}0 & \text { if } x \leq 5 \\ 1 & \text { if } x>5\end{cases}
$$

Clearly, $f$ is an onto $R$-semimodule homomorphism. Also $\operatorname{ker} f=\{0,1,2,3,4,5\}$ is a partitioning subsemimodule of $M$ with $Q=\{0,6,7, \ldots\}$. For $1 \in M^{\prime}$ there cannot exists any $q_{1} \in f^{-1}(\{1\})$ such that $x+\operatorname{ker} f \subseteq q_{1}+\operatorname{ker} f$ for all $x \in f^{-1}(\{1\})$. So $f$ is not a maximal $R$-semimodule homomorphism.

Example 2.10. Let $M=\left(\mathbb{Z}_{0}^{+},+\right), M^{\prime}=\left(\mathbb{Z}_{6},{ }_{6}\right)$ and $R=\left(\mathbb{Z}_{0}^{+},+, \cdot\right)$. Then $M$, $M^{\prime}$ are $R$-semimodules. Define $f: M \rightarrow M^{\prime}$ by $f(x)=\bar{r}$ where $x \equiv r(\bmod$ $6), 0 \leq r \leq 5$. Clearly, $f$ is onto $R$-semimodule homomorphism. Also ker $f=$ $\{0,6,12,18, \ldots\}$. For any $\bar{a} \in M^{\prime}$ there exists a unique $q_{a}=a \in f^{-1}(\{\bar{a}\})$ such that $x+\operatorname{ker} f \subseteq q_{a}+\operatorname{ker} f$ for all $x \in f^{-1}(\{\bar{a}\})$. Hence $f$ is a maximal $R$-semimodule homomorphism.

Lemma 2.11. Let $M, M^{\prime}, f$ and $Q$ be as stated in Lemma 2.8. Let $q_{a}, q_{b}, q_{c} \in Q$ and $r \in R$, then

(i) If $q_{a}+q_{b}+\operatorname{kerf} \subseteq q_{c}+\operatorname{kerf} f$, then $a+b=c$.

(ii) If $r q_{a}+k e r f \subseteq r q_{c}+k e r f$, then $r a=r c$.

Proof. (i) Since $q_{a}+q_{b} \in q_{a}+q_{b}+\operatorname{ker} f \subseteq q_{c}+\operatorname{kerf}, q_{a}+q_{b}=q_{c}+k$ for some $k \in$ kerf. Now $a+b=f\left(q_{a}\right)+f\left(q_{b}\right)=f\left(q_{a}+q_{b}\right)=f\left(q_{c}+k\right)=f\left(q_{c}\right)+f(k)=c$. (ii) Since $r q_{a} \in r q_{a}+k e r f \subseteq r q_{c}+k e r f, r q_{a}=r q_{c}+k^{\prime}$ for some $k^{\prime} \in k e r f$. Now $r a=r f\left(q_{a}\right)=f\left(r q_{a}\right)=f\left(r q_{c}+k^{\prime}\right)=f\left(r q_{c}\right)+f\left(k^{\prime}\right)=r f\left(q_{c}\right)=r c$

Theorem 2.12. If $f: M \rightarrow M^{\prime}$ is a maximal $R$-semimodule homomorphism, then $M / \operatorname{ker} f_{(Q)} \cong M^{\prime}$ where $Q$ is as stated in Lemma 2.8.

Proof. By Lemma 2.8, $\operatorname{kerf}$ is a partitioning subsemimodule of $M$. Define $\bar{f}$ : $M / \operatorname{kerf}_{(Q)} \rightarrow M^{\prime}$ by $\bar{f}\left(q_{a}+\operatorname{kerf}\right)=f\left(q_{a}\right)=a$ for each $q_{a} \in Q$. If $q_{a}+\operatorname{kerf}, q_{b}+$ $\operatorname{kerf} \in M / \operatorname{ker} f_{(Q)}$, then $\bar{f}\left(q_{a}+\operatorname{kerf}\right)=\bar{f}\left(q_{b}+\operatorname{kerf}\right) \Leftrightarrow a=b \Leftrightarrow q_{a}+\operatorname{kerf}=q_{b}$ $+\operatorname{ker} f$. Hence $\bar{f}$ is well defined and one-one. Since $f$ is maximal, $\bar{f}$ is onto. For $q_{a}$ $+\operatorname{kerf}, q_{b}+\operatorname{kerf} \in M / \operatorname{kerf} f_{(Q)}, r \in R$, consider (i) $\bar{f}\left(\left(q_{a}+\operatorname{kerf}\right) \oplus\left(q_{b}+\operatorname{kerf}\right)\right)$ 
$=\bar{f}\left(q_{c}+\operatorname{ker} f\right)=c$ where $q_{c}$ is a unique element in $Q$ such that $q_{a}+q_{b}+k e r f \subseteq$ $q_{c}+$ kerf. By Lemma 2.11, $a+b=c$. Now $\bar{f}\left(q_{a}+\right.$ kerf $)+\bar{f}\left(q_{b}+\right.$ kerf $)=a+$ $b=c=\bar{f}\left(\left(q_{a}+\operatorname{ker} f\right) \oplus\left(q_{b}+\operatorname{ker} f\right)\right)$. (ii) $\bar{f}\left(r \odot\left(q_{a}+\operatorname{ker} f\right)\right)=\bar{f}\left(q_{d}+\operatorname{kerf}\right)=d$ where $q_{d}$ is a unique element in $Q$ such that $r q_{a}+k e r f \subseteq q_{d}+k e r f$. By Lemma $2.11, r a=d$. Therefore, $r \bar{f}\left(q_{a}+k e r f\right)=r a=d=\bar{f}\left(r \odot\left(q_{a}+k e r f\right)\right)$. Hence $\bar{f}$ is an $R$-semimodule isomorphism. Thus, $M / \operatorname{ker} f_{(Q)} \cong M^{\prime}$.

\section{Subtractive subsemimodules}

In this section we extend some results of S. E. Atani [4] to semimodules over semirings.

Definition 3.1. A subsemimodule $N$ of an $R$-semimodule $M$ is called a subtractive subsemimodule (=k-subsemimodule) if $x, x+y \in N, y \in M$ then $y \in N$.

Theorem 3.2. Every partitioning subsemimodule $N$ of an $R$-semimodule $M$ is subtractive.

Proof. Since $N$ is a partitioning subsemimodule, by Lemma 2.3, $N=q_{0}+N$ for some $q_{0} \in Q$. Let $x, x+y \in N$ where $y \in M$. Therefore $x=q_{0}+\alpha, x+y=q_{0}+$ $\beta$ for some $\alpha, \beta \in N$. By Lemma 2.2, there exists a unique $q^{\prime} \in Q$ such that $y=q^{\prime}$ $+\lambda$ for some $\lambda \in N$. Now $y+x=q^{\prime}+\lambda+x \in q^{\prime}+N$ and $x+y \in N=q_{0}+N$. Hence $\left(q_{0}+N\right) \cap\left(q^{\prime}+N\right) \neq \emptyset$ and so $q_{0}=q^{\prime}$. Thus, $y=q^{\prime}+\lambda \in q^{\prime}+N=q_{0}$ $+N=N$.

If $(M,+)$ is an idempotent commutative monoid, then $M$ is $\left(\mathbb{Z}_{0}^{+},+, \cdot\right)$ semimodule with scalar multiplication defined by $r m=0$ if $r=0$ and $r m=m$ if $r>0$ for all $r \in \mathbb{Z}_{0}^{+}$and $m \in M$ ([5], P.151). In a semiring $R=\left(\mathbb{Z}_{0}^{+}, \mathrm{gcd}, l \mathrm{~cm}\right)$, the ideal $2 \mathbb{Z}_{0}^{+}=\{0,2,4,6, \ldots\}$ of $R$ is subtractive but not partitioning ([7]).

The converse of Theorem 3.2 is not true.

Example 3.3. Let $M=\left(\mathbb{Z}_{0}^{+}\right.$, gcd $)$and $R=\left(\mathbb{Z}_{0}^{+},+, \cdot\right)$. Clearly, $M$ is a commutative monoid in which every element is idempotent. Hence $M$ is an $R$ semimodule in which $N=\{0,2,4,6, \ldots\}$ is a subtractive subsemimodule of $M$ but not a partitioning subsemimodule.

S. E. Atani ([4], Lemma 2.1, Proposition 2.2 and Theorem 2.3) has proved the results for semirings. However, we extend the following Lemma 3.4, Theorem 3.5 and Theorem 3.6 for semimodules over semirings.

Lemma 3.4. Let $N$ be a $Q$-subsemimodule of an $R$-semimodule $M$. If $A$ is a subtractive subsemimodule of $M$ such that $N \subseteq A$, then $N$ is a $Q \cap A$-subsemimodule of $A$.

Proof. It is sufficient to show that $A=\cup\{q+N: q \in Q \cap A\}$. Clearly, $\cup\{q+N$ : $q \in Q \cap A\} \subseteq A$. On the other hand, let $x \in A$. Since $N$ is a $Q$-subsemimodule, by Lemma 2.2, $x=q+a$ for some $q \in Q, a \in N \subseteq A$. Then $q \in Q \cap A$, since $A$ is a 
subtractive subsemimodule. So we have an equality.

Theorem 3.5. Let $N$ be a $Q$-subsemimodule, $A$ be a subtractive subsemimodule of an R-semimodule $M$ with $N \subseteq A$. Then $A / N_{(Q \cap A)}=\{q+N: q \in Q \cap A\}$ is a subtractive subsemimodule of $M / N_{(Q)}$.

Proof. By Lemma 2.3, let $q_{0} \in Q$ be unique such that $q_{0}+N$ is the zero element of $M / N_{(Q)}$. First, we show that $q_{0}+N \in A / N_{(Q \cap A)}$. Let $a+N \in A / N_{(Q \cap A)}$ $\subseteq M / N_{(Q)}$ where $a \in Q \cap A$. Then $(a+N) \oplus\left(q_{0}+N\right)=a+N$ where $a \in Q$ is a unique such that $a+q_{0}+N \subseteq a+N$. So $a+q_{0}=a+b$ for some $b \in N \subseteq A$. Since $A$ is a subtractive subsemimodule, $q_{0} \in A$. Thus, $q_{0}+N \in A / N_{(Q \cap A)}$. Next suppose that $q_{1}+N, q_{2}+N \in A / N_{(Q \cap A)}$ where $q_{1}, q_{2} \in Q \cap A$. Then $\left(q_{1}+N\right) \oplus\left(q_{2}+N\right)=$ $q_{3}+N$ where $q_{3} \in Q$ is a unique such that $q_{1}+q_{2}+N \subseteq q_{3}+N$. So $q_{1}+q_{2}=q_{3}+c$ for some $c \in N \subseteq A$. Hence $q_{3} \in Q \cap A$, since $A$ is a subtractive subsemimodule. Now $\left(q_{1}+N\right) \oplus\left(q_{2}+N\right)=q_{3}+N \in A / N_{(Q \cap A)}$. Now let $r \in R, q+N \in A / N_{(Q \cap A)}$. Then $r$ $\odot(q+N)=q_{4}+N$ where $q_{4} \in Q$ is a unique such that $r q+N \subseteq q_{4}+N$. So $r q=q_{4}+d$ for some $d \in N \subseteq A$. Hence $q_{4} \in Q \cap A$, since $A$ is a subtractive subsemimodule. Thus, $r \odot(q+N)=q_{4}+N \in A / N_{(Q \cap A)}$. Thus $A / N_{(Q \cap A)}$ is a subsemimodule of $M / N_{(Q)}$. Finally, assume that $x+N,(x+N) \oplus(y+N)=z+N \in A / N_{(Q \cap A)}$ where $x, z \in Q \cap A, y \in Q$ and $x+y+N \subseteq z+N$. Then $x+y=z+e$ for some $e \in N \subseteq A$. Now $y \in Q \cap A$, since $A$ is a subtractive subsemimodule. Thus $y+N \in A / N_{(Q \cap A)}$ as needed.

Theorem 3.6. Let $N$ be a $Q$-subsemimodule of an $R$-semimodule $M$ and $L$ be a subtractive subsemimodule of $M / N_{(Q)}$. Then $L=P / N_{(Q \cap P)}$ for some subtractive subsemimodule $P$ of $M$ with $N \subseteq P$.

Proof. By Lemma 2.2, let $q_{0}+N=N$ be the zero element of $M / N_{(Q)}$ where $q_{0} \in Q$. Denote $P=\{x \in M$ : there exists a unique $q \in Q$ such that $x+N \subseteq$ $q+N \in L\}$. We show that $P$ is a subtractive subsemimodule of $M$ with $N \subseteq P$ and $L=P / N_{(Q \cap P)}$.

(1) Let $x \in N$. Then $x+N \subseteq N=q_{0}+N \in L$, so $x \in P$. Thus, $N \subseteq P$.

(2) Let $x, y \in P$. Then there are unique elements $q_{1}, q_{2} \in Q$ such that $x+N \subseteq$ $q_{1}+N \in L$ and $y+N \subseteq q_{2}+N \in L$. Now $\left(q_{1}+N\right) \oplus\left(q_{2}+N\right)=q_{3}+N \in$ $L$ where $q_{3} \in Q$ is a unique such that $q_{1}+q_{2}+N \subseteq q_{3}+N$. By Lemma 1.3, $x+$ $N \subseteq q_{1}+N$ and $y+N \subseteq q_{2}+N$ implies $x+y+N \subseteq q_{1}+y+N \subseteq q_{1}+q_{2}+N$ $\subseteq q_{3}+N \in L$. Hence $x+y \in P$. Similarly, if $r \in R, x \in P$ then $r x \in P$. Thus, $P$ is a subsemimodule of $M$.

(3) Let $x, x+y \in P$ where $y \in M$. Then there are unique elements $q_{1}, q_{2}, q_{3} \in Q$ such that $x+N \subseteq q_{1}+N \in L, x+y+N \subseteq q_{2}+N \in L, y+N \subseteq q_{3}+N \in M / N_{(Q)}$. Since $q_{1}+N, q_{3}+N \in M / N_{(Q)}$, there exists a unique element $q_{4} \in Q$ such that $\left(q_{1}+N\right) \oplus\left(q_{3}+N\right)=q_{4}+N$ where $q_{1}+q_{3}+N \subseteq q_{4}+N$. By Lemma 1.3, $x+N \subseteq$ $q_{1}+N$ and $y+N \subseteq q_{3}+N$ implies $x+y+N \subseteq q_{1}+y+N \subseteq q_{1}+q_{3}+N \subseteq q_{4}+N$. Hence $x+y \in\left(q_{2}+N\right) \cap\left(q_{4}+N\right)$. So $q_{4}+N=q_{2}+N \in L$. Since $L$ is a subtractive subsemimodule, $q_{3}+N \in L$. Now $y+N \subseteq q_{3}+N \in L$. So $y \in P$. Hence $P$ is a subtractive subsemimodule of $M$. 
(4) By Lemma 3.4, $N$ is a $Q \cap P$-subsemimodule of $P$. If $q+N \in L$ where $q \in Q$ then $q \in P$. So $q \in Q \cap P$, hence $q+N \in P / N_{(Q \cap P)}$. Thus, $L \subseteq P / N_{(Q \cap P)}$. On the other hand, if $q+N \in P / N_{(Q \cap P)}$, then $q \in Q \cap P \subseteq P$. So $q+N \subseteq q^{\prime}+N \in L$ for some unique $q^{\prime} \in Q$. Therefore, $q+N=q^{\prime}+N \in L$. Thus, $P / N_{(Q \cap P)} \subseteq L$

The authors are thankful to the referee for his helpful suggestions.

\section{References}

[1] Paul J. Allen, A fundamental theorem of homomorphism for semirings, Proc. Amer. Math. Soc., 21(1969), 412-416.

[2] Paul J. Allen, J. Neggers and H. S. Kim, Ideal theory in commutative A-semirings, Kyungpook. Math. Journal, 46(2006), 261-271.

[3] R. E. Atani and S. E. Atani, On subsemimodules of semimodules, Buletinul Acad. Sci. Republ. Moldova, ser. Math., to appear (2010).

[4] Shahabaddin Ebrahimi Atani, The ideal theory in quotient of commutative semirings, Glasnik Matematicki, Vol. 42(62)(2007), 301-308.

[5] J. S. Golan, Semiring and their Applications, Kluwer Academic publisher Dordrecht, 1999.

[6] Vishnu Gupta and J. N. Chaudhari, On Right $\pi$-Regular Semirings, Sarajevo Journal of Mathematics. Vol 2(14)(2006), 3-9.

[7] Vishnu Gupta and J. N. Chaudhari, On Partitioning ideals of Semirings, Kyungpook. Math. Journal, 46(2006), 181-184.

[8] Vishnu Gupta and J. N. Chaudhari, Some remarks on semirings, Radovi Matematicki, 12(2003), 13-18. 